\title{
Spindle Cell Hemangioendothelioma
}

\author{
A Low-Grade Angiosarcoma Resembling a Cavernous \\ Hemangioma and Kaposi's Sarcoma
}

\author{
Sharon W. Weiss, M.D., and F. M. Enzinger, M.D.
}

\begin{abstract}
Twenty-six cases of a newly recognized form of vascular tumor are presented. The tumor may occur at any age, has a male predominance, and develops preferentially in the dermis and subcutaneous tissues of the distal extremities. Histologically it combines the features of both a cavernous hemangioma and Kaposi's sarcoma. It is composed of thin-walled cavernous vessels which may be dilated, partially collapsed, or filled with organizing thrombi and phleboliths. These areas are intimately associated with spindled areas reminiscent of Kaposi's sarcoma. The spindled areas differ from Kaposi's sarcoma by the presence of occasional epithelioid endothelial cells, which sometimes display vacuolization. Follow-up information in 14 cases indicates that nine patients experienced "recurrences." One patient, who also received radiotherapy, developed regional lymph node metastasis 40 years after diagnosis and following 19 recurrences. No patient, however, has died of his disease, despite relatively limited surgical excision. The term "spindle cell hemangioendothelioma" is suggested for this vascular tumor of low-grade malignancy.
\end{abstract}

Am J Surg Pathol 10(8): 521-530, 1986.

From the Department of Soft Tissue Pathology, Armed Forces Institute of Pathology, Washington, D.C.

The opinions or assertions contained herein are the private views of the authors and are not to be construed as official or as reflecting the views of the Department of the Army or Defense. Address correspondence and reprint requests to Sharon W. Weiss, M.D., Department of Soft Tissue Pathology, Armed Forces Institute of Pathology, Washington, D.C. 20306-6000, U.S.A.
In the process of reviewing a large number of angiosarcomas, we have identified a small and hitherto unrecognized subset of vascular tumors that combine the features of both a cavernous hemangioma and Kaposi's sarcoma. Because most of these tumors grow in a slowly progressive fashion with eventual involvement of a significant area of the body, we suggest that they are a form of lowgrade angiosarcoma and propose the term "spindle cell hemangioendothelioma."

\section{MATERIALS AND METHODS}

The 26 cases comprising this study represented consultation material submitted to the Armed Forces Institute of Pathology during the years 1955 to 1985 . The first six cases were ascertained as a result of a retrospective review of several hundred angiosarcomas of skin and soft tissue, and the remainder of the cases were obtained prospectively because of familiarity with the characteristics of this unusual lesion. In all cases, hematoxylineosin-stained slides were examined. Immunohistochemical stains for factor VIII-associated antigen were performed by the peroxidase-antiperoxidase technique according to the method of Sternberger. Full elucidation of the antigen required prior enzymatic digestion of tissue sections.

Electron microscopy was performed in two cases from fresh tissue fixed in glutaraldehyde and postfixed in osmium tetroxide. Thick sections $(0.5-1.5$ $\mu \mathrm{m})$ were stained with toluidine blue, and thin sections were stained with uranyl acetate and lead citrate and examined with a Zeiss transmission electron microscope.

Steroid receptor analysis was performed on two cases on fresh tissue by means of dextran-coated charcoal method (Herner Analyticals, Bethesda, MD). This included receptor protein determina- 
tions for estrogen, progesterone, glucocorticoid, and testosterone.

\section{CLINICAL FINDINGS}

As indicated in Table 1, patients with these tumors may present at any age. However, about half of patients indicated that the first lesion(s) was noted before the age of 25 years, and in two of these patients the lesions were allegedly noted shortly after birth. The long interval between onset of a lesion and hospital admission in many cases serves to emphasize the slow progression of the lesion. In fact, four patients postponed seeking medical advice for over 30 years. Seventeen patients were male and nine female. The most commonly affected site was the hand (12 cases) followed by foot (six cases), chest wall (four cases), arm (two cases), leg (two cases), and abdominal wall (one case). In two patients lesions were present on both arm and chest wall, accounting for double counting in the foregoing figures. The age, sex, and location were unknown in one case.

The most common presentation was that of one or more painless nodules that slowly increased in size or number over a period of several months or a few years. In 17 patients a complete clinical description of the original lesion was available. In 10 cases the initial lesion was solitary and in seven multiple. Of multiple lesions, all occurred in the same general area, except for one patient who had bilateral lesions of the hands. In four of the 17 patients a definite history of progression from one to several nodules was elicited. All but two cases were located in the superficial soft tissue (e.g., dermis, subcutis) and therefore were diagnosed clinically as various benign dermatologic lesions such as epidermal inclusion cyst, sebaceous cyst, hemangioma, or dermatofibroma. Two lesions located in deep soft tissue produced no specific symptoms. One lesion from the axilla presented as a large mass closely attached to the brachial plexus,

TABLE 1. Age distribution at presentation with spindle cell hemangioendothelioma

\begin{tabular}{lc}
\hline Age $(y r)$ & Patients $(n)$ \\
\hline $0-10$ & 1 \\
$11-20$ & 4 \\
$21-30$ & 5 \\
$31-40$ & 5 \\
$41-50$ & 6 \\
$51-60$ & 1 \\
$>60$ & 3 \\
Unknown & 1 \\
Total & 26 \\
\hline
\end{tabular}

whereas the second case, a recurrent lesion, consisted of small nodules which involved the subcutis and muscles of the entire lower leg. Computed tomography showed numerous soft tissue densities within the lower density fat (Fig. 1). Some of the soft tissue densities had a central high-density core corresponding to the presence of phleboliths.

Three patients had interesting and pertinent associated clinical findings: The first had a history of Milroy's disease and developed the lesion within an area of chronic lymphedema. The second had Mafucci's syndrome and had multiple spindle cell hemangioendotheliomas $(\mathrm{SCH})$ of the arm and chest in association with a high-grade angiosarcoma of the abdominal wall. The third patient developed several SCHs in the immediate vicinity of an epithelioid hemangioendothelioma, which had been present for several decades.

\section{GROSS AND MICROSCOPIC APPEARANCE}

Grossly the tumors were small, red, hemorrhagic nodules, which appeared well circumscribed and varied in size from a few millimeters to $4 \mathrm{~cm}$ in diameter (Fig. 2). Some contained pearly-white phleboliths that "popped out" when the tumors were sectioned. In "recurrent" lesions the nodules of tumor were often located several centimeters from one another in a "skip" pattern.

All the tumors were composed of two components: cavernous endothelial-lined blood spaces and cellular zones composed of a less differentiated spindle cell component (Figs. 3 and 4). The relative amounts of these components varied from lesion to lesion and from case to case. A typical lesion was composed of small nodules in the dermis or subcutis which seemed well demarcated but which
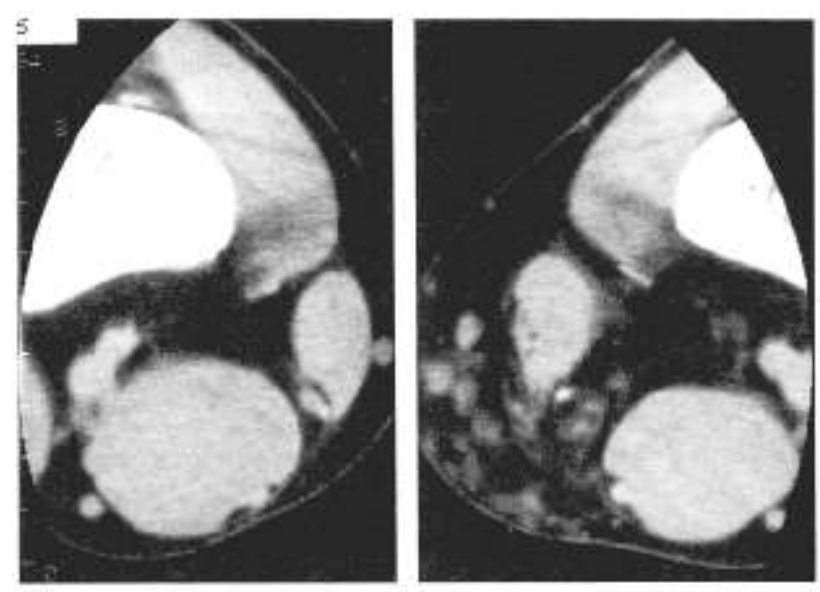

FIG. 1. CT scan showing numerous soft tissue densities involving subcutaneous fat and muscle of thigh (right) compared to normal (left) side. 
(a)

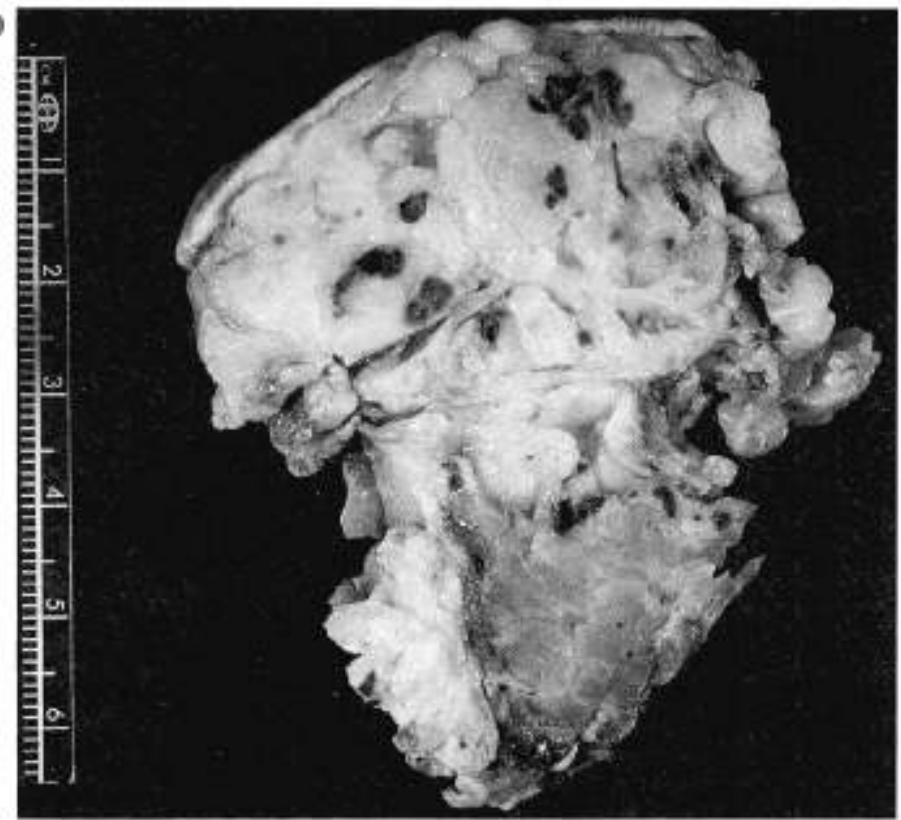

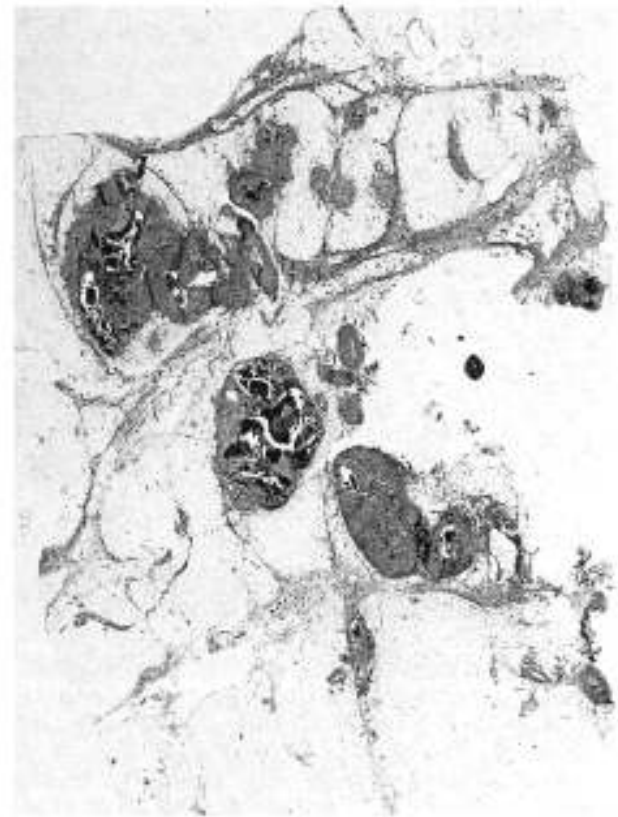

(b)

FIG. 2. (a) Gross specimen corresponding to area displayed in Figure 1. Numerous punctate hemorrhagic tumor nodules involve fat and superficial areas of muscle. (b) Low-power view of subcutaneous fat from (a) illustrating myriad tumor nodules of varying sizes.

gave rise to tiny capillary sprouts at their periphery. A reactive myxoid zone with lymphocytes partially surrounded the nodules. In two cases the original lesion appeared to arise within a vessel (Fig. 5). At low power the lesions were typically mistaken for a cavernous hemangioma by virtue of the large, di-

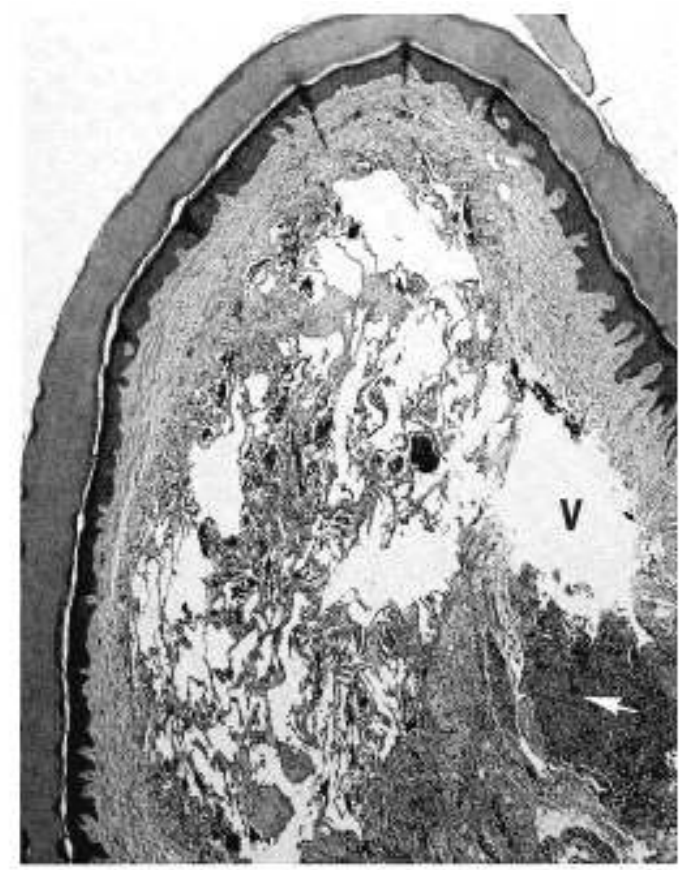

FIG. 3. Low-power view of tumor illustrating regional variation of tumor. Solidly cellular (white arrow) areas of tumor contrast with vascular areas (V). lated, or partially collapsed vessels (Fig. 6), which were filled with blood, thrombi, or phleboliths (Fig. 4). Usually the walls of these vessels were devoid of muscle and contained only small amounts of collagen. Scattered between the gaping vessels was a population of spindled cells, some of which lined

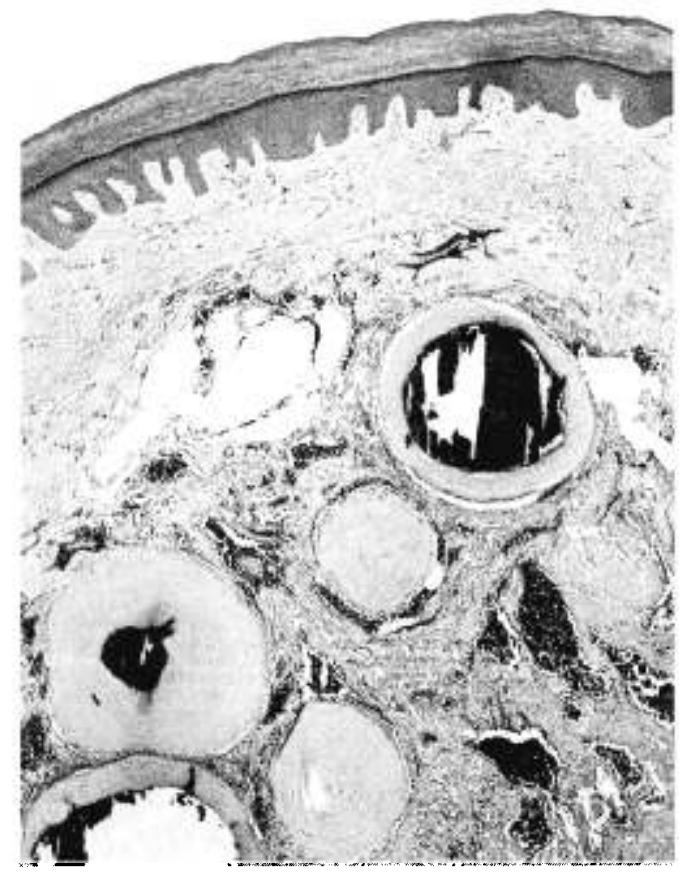

FIG. 4. Low-power view of tumor showing phleboliths within cavernous vascular spaces. 


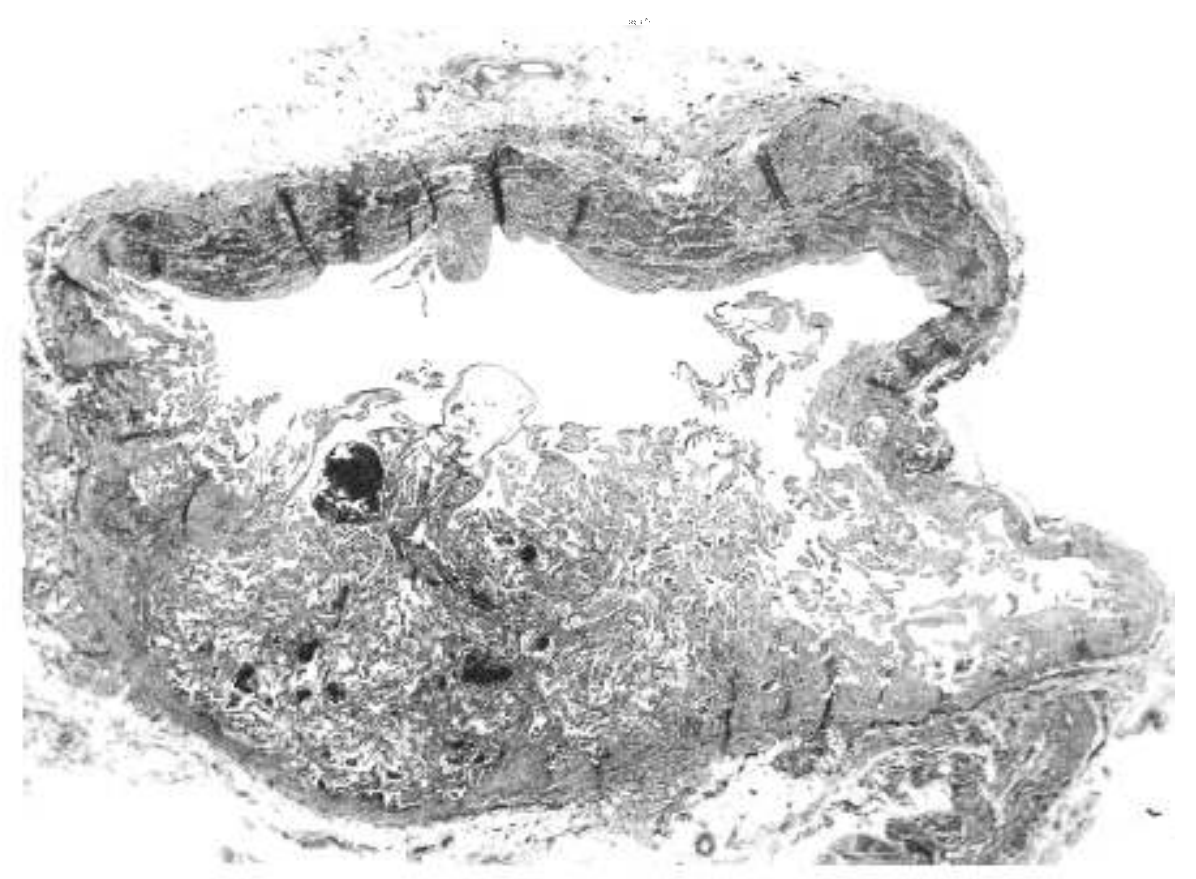

FIG. 5. Spindle cell hemangioendothelioma arising within a vessel. slit-like vascular spaces (Fig. 7). Others, however, were arranged in short fascicles and were not intimately related to vascular channels. In the majority of cases the spindle cells were well differentiated in that nuclear atypia was minimal. In 24 of 26 cases mitotic figures were not detected, and in two cases they were present at low levels (less than $1 / 10$ highpower fields). Although the spindled areas, out of context, resembled Kaposi's sarcoma, they differed principally by the focal presence of plump or rounded endothelial cells. The latter cells imparted a "biphasic" appearance to the spindled zones (Fig. 8). These plump endothelial cells could be seen as small nests or sometimes lining vascular channels. They had a clear cytoplasm which occasionally displayed "vacuolar" change, which is seen in various endothelial tumors and which is generally regarded as miniature intracytoplasmic lumen formation by a single cell. In several cases the vacuolar change of the endothelium was so prominent that the cells could easily be mistaken for a fat cell or lipoblast on cursory inspection (Fig.

FIG. 6. Characteristic appearance of tumor depicting intermingling of cavernous spaces and spindle cell areas.

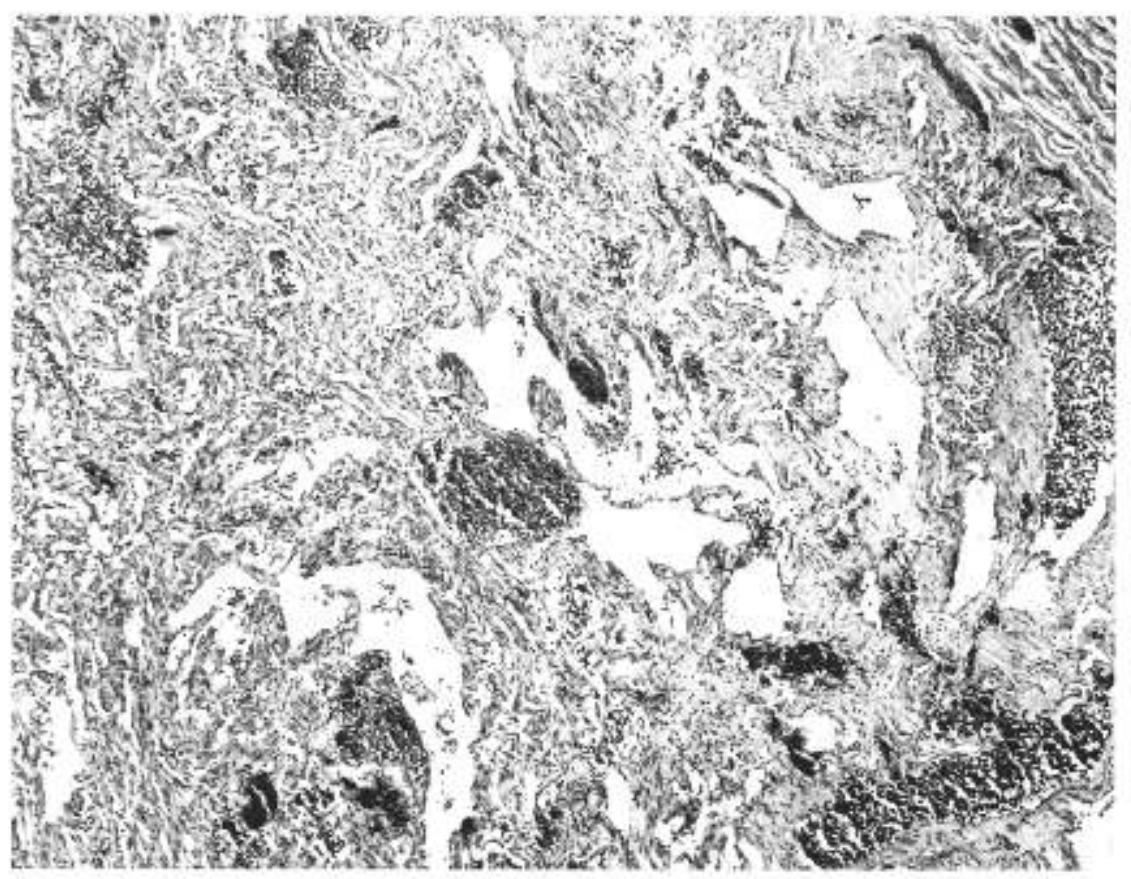


(a)

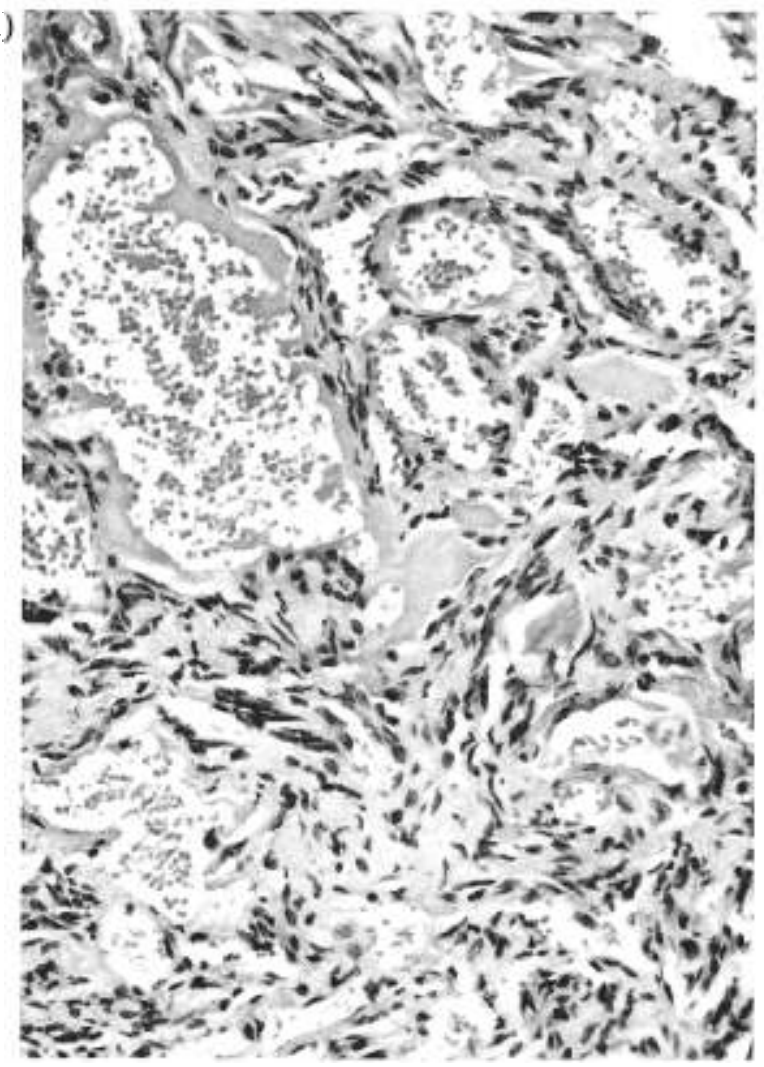

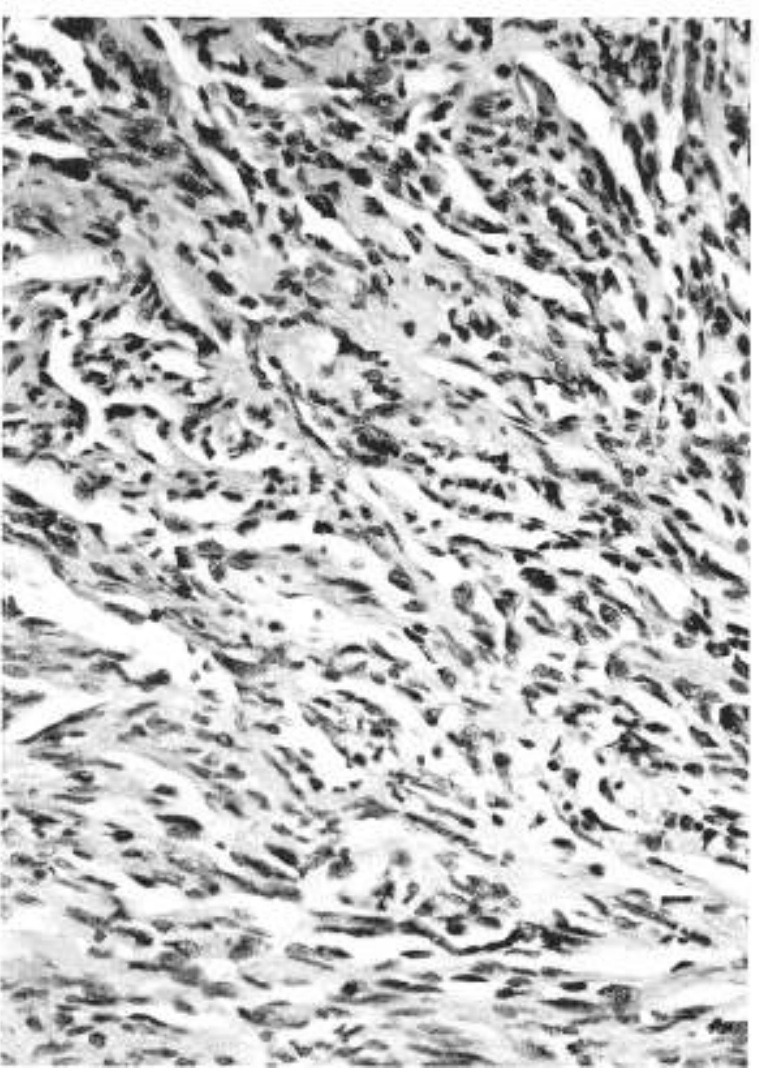

FIG. 7. (a) Cavernous blood spaces with intervening spindled cells. (b) Cellular areas with vascular slits.

8 b). Recurrent lesions were similar to the original lesion. However, in the one case that eventually metastasized, the nineteenth recurrence displayed pleomorphism and mitotic activity.

\section{ULSTRASTRUCTURAL FINDINGS}

The cells lining well-formed vascular channels possessed many of the features of normal endothelial cells (Fig. 9). They rested on a well-developed, complete, and sometimes reduplicated basal lamina; they formed elaborate interdigitations and junctional complexes with neighboring cells. At their surface they possessed small projections and at their antiluminal border numerous pinocytotic vesicles. Occasional Weibel-Palade bodies and hemosiderin granules were present within their cytoplasm. Some of the cells were filled with collections of intermediate filaments (Fig. 9). Others also contained small intracytoplasmic lumina, corresponding to the vacuolization noted by light microscopy. Cells that did not line blood spaces were more difficult to characterize. Some were clearly pericytes in that they bore a consistent topographical relationship to the endothelial cells, were partially invested with basal lamina and contained pinocytotic vesicles. Others appeared rounded or slightly spindled. They were neither related to endothelial cells nor invested by basal lamina, yet their frequent intimate association with one another in small cohesive groups suggested partial endothelial differentiation (Fig. 10). Organelles were sparse within the cytoplasm, although occasional pinocytotic vesicles were noted along the surfaces. Still other cells resembled primitive mesenchymal cells (Fig. 11). They had a stellate or spindled shape, high nuclear:cytoplasmic ratio, and few organelles.

\section{IMMUNOHISTOCHEMICAL FINDINGS}

Antibody to factor VIII-associated antigen decorated most of the endothelial cells lining the cavernous blood spaces. Immunoreactive factor VIIIassociated protein was also demonstrated within occasional rounded endothelial cells, which either lined small vessels or lay in solid groups within the spindled stroma. Unequivocal factor VIII-associated protein was rarely, if ever, identified within the spindled cells.

\section{STEROID RECEPTOR ANALYSIS}

Estrogen and progesterone receptor analysis was negative in the two cases studied. However, an- 
(a)

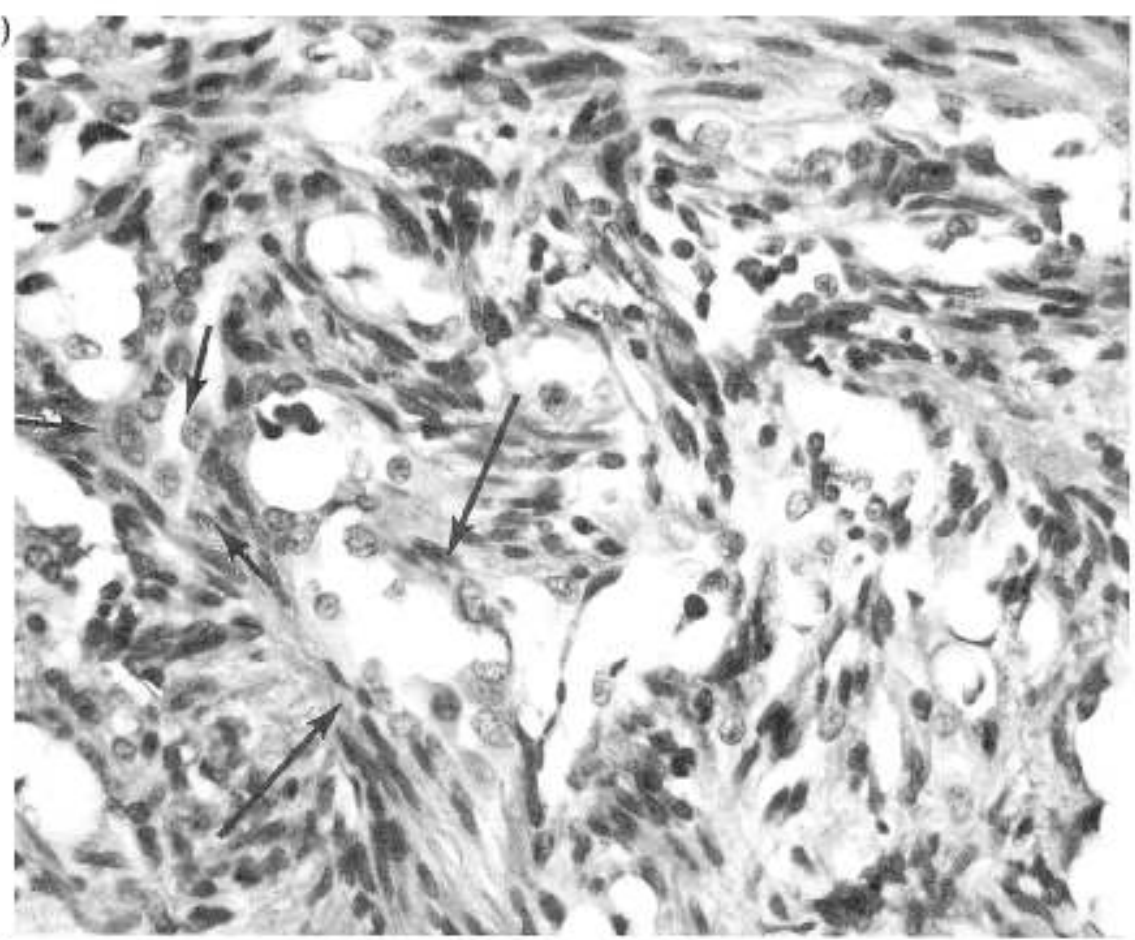

(b)

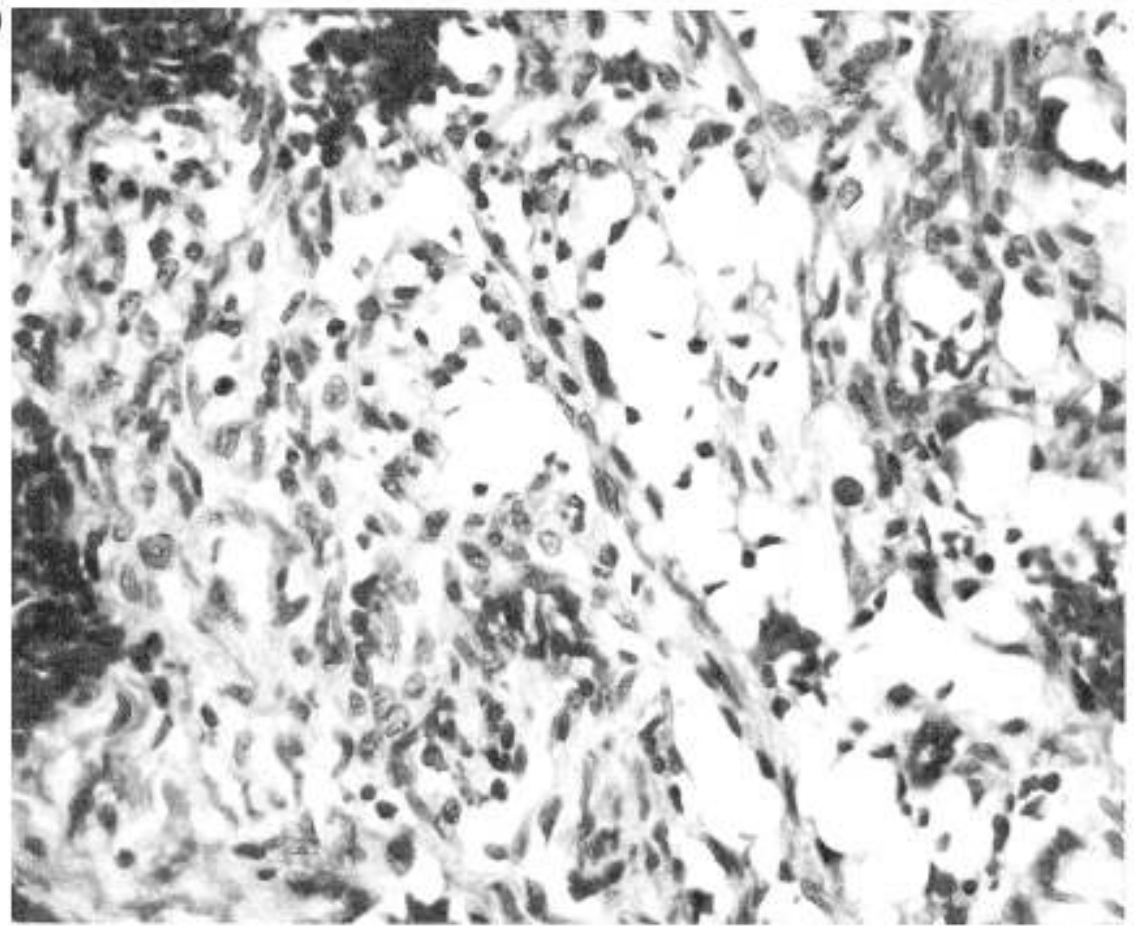

FIG. 8. (a) Cellular spindle areas illustrating focal epithelioid endothelial cells (short arrows), which lie in small groups and also line vascular channels (long arrows). (b) Markedly vacuolated endothelial cells, which are often confused with fat cells or lipoblasts. drogen and glucocorticoid receptor was detected at levels of 47 and $1272 \mathrm{fmol} / \mathrm{mg}$ protein respectively within the abdominal wall tumor of a 21-year-old male. In the second case glucocorticoid receptor was present in the wrist lesion $(275 \mathrm{fmol} / \mathrm{mg})$ of a 24-year-old man.

\section{TREATMENT AND BEHAVIOR}

Follow-up information was available for 14 of 26 patients; the median follow-up was between 6 and 8 years, and the average follow up was 14 years. In this group 10 patients experienced local recur- 


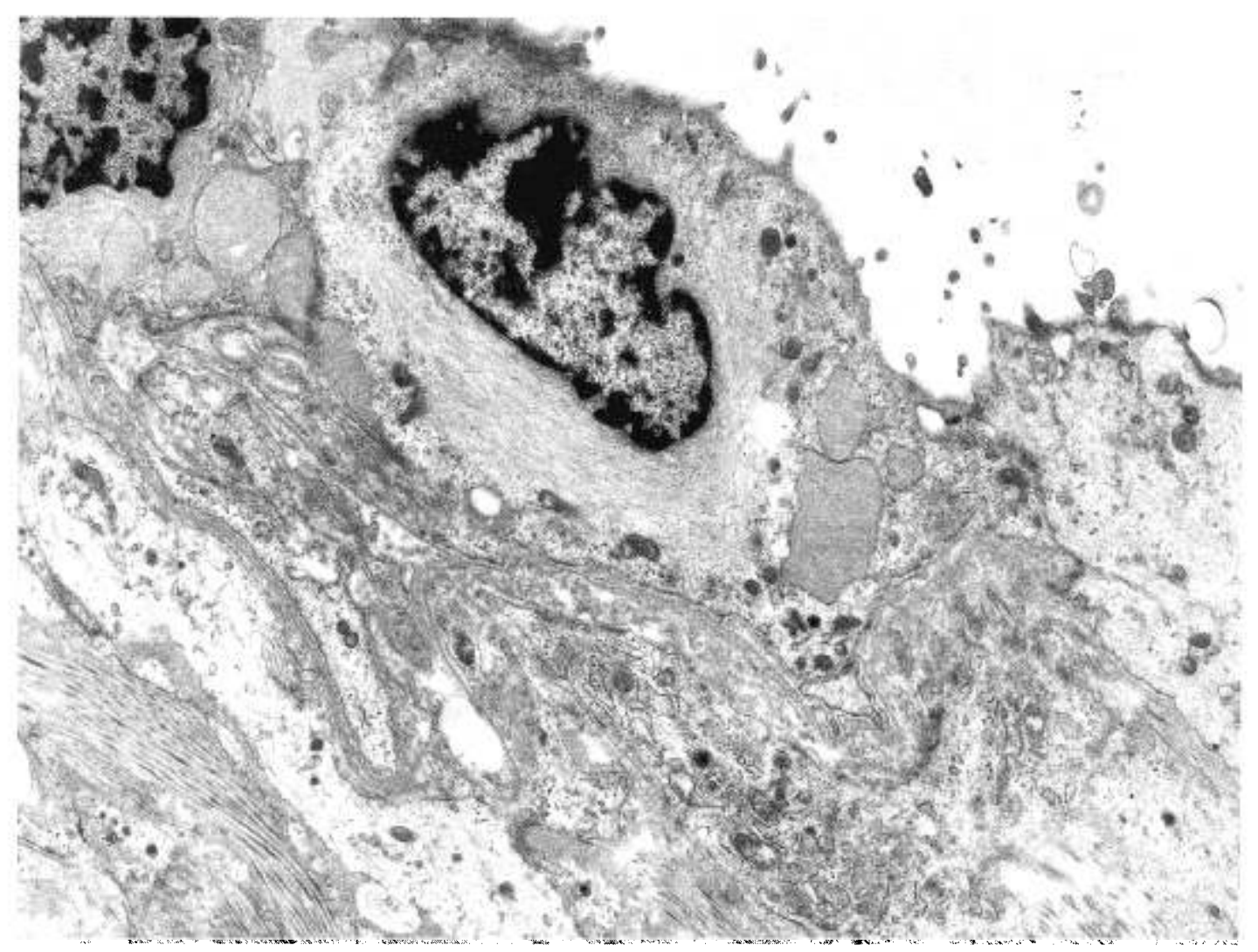

FIG. 9. Electron micrograph of well-differentiated endothelial cell lining vascular space. Cell contains abundant cytoplasmic filaments, probably vimentin. Complete, well-formed basal lamina is present on antiluminal border.

rences; seven of these had one recurrence, two had two recurrences, and one had 19 recurrences over a 40 -year period. All patients experiencing recurrences had initial simple excisions; four subsequently underwent wide excision, and one had radiotherapy for control of local disease. Recurrences developed from 2 to 40 years following the initial therapy. In general, recurrences consisted of two or more nodules in the vicinity of the original tumor. However, in two cases the recurrent lesions recurred so far proximal to the original lesion that the possibility of skip metastases or multifocal disease was considered. In one of these two patients, recurrent tumor extensively involved the subcutis and superficial muscles of the leg (Figs. 1 and 2) to the level of the groin, even though the original mass was located in the ankle. Debulking of tumor was carried out and the patient is currently alive with residual disease. In the second case, the original tumor was situated in the distal arm, and the two recurrences involved the upper arm and chest wall respectively. In only one of the 13 cases did unequivocal metastatic disease develop. This patient, experiencing 19 recurrences over a 40-year period, ultimately developed a regional lymph node metas- tasis. Evaluation of this case was complicated by the fact that some of the lesions had been irradiated and the last recurrence had areas resembling a conventional high-grade angiosarcoma.

\section{DISCUSSION}

The vascular tumors described in this paper represent a new and previously unrecognized subset of low-grade angiosarcoma culled from a review of several hundred vascular lesions at our institution. Patients with spindle cell hemangioendothelioma (SCH) typically develop one or more lesions in the distal portion of the extremities. Those involving the dermis can easily be confused clinically with a hemangioma or Kaposi's sarcoma, whereas those in the subcutis are clinically nondistinctive. The hallmarks of this tumor are the peculiar juxtaposition of cavernous blood spaces and a spindle cell stroma. The two components vary quantitatively from lesion to lesion and account for the range of diagnoses that are often entertained (e.g., hemangioma, arteriovenous hemangioma, Kaposi's sarcoma).

Although these cases are better differentiated 


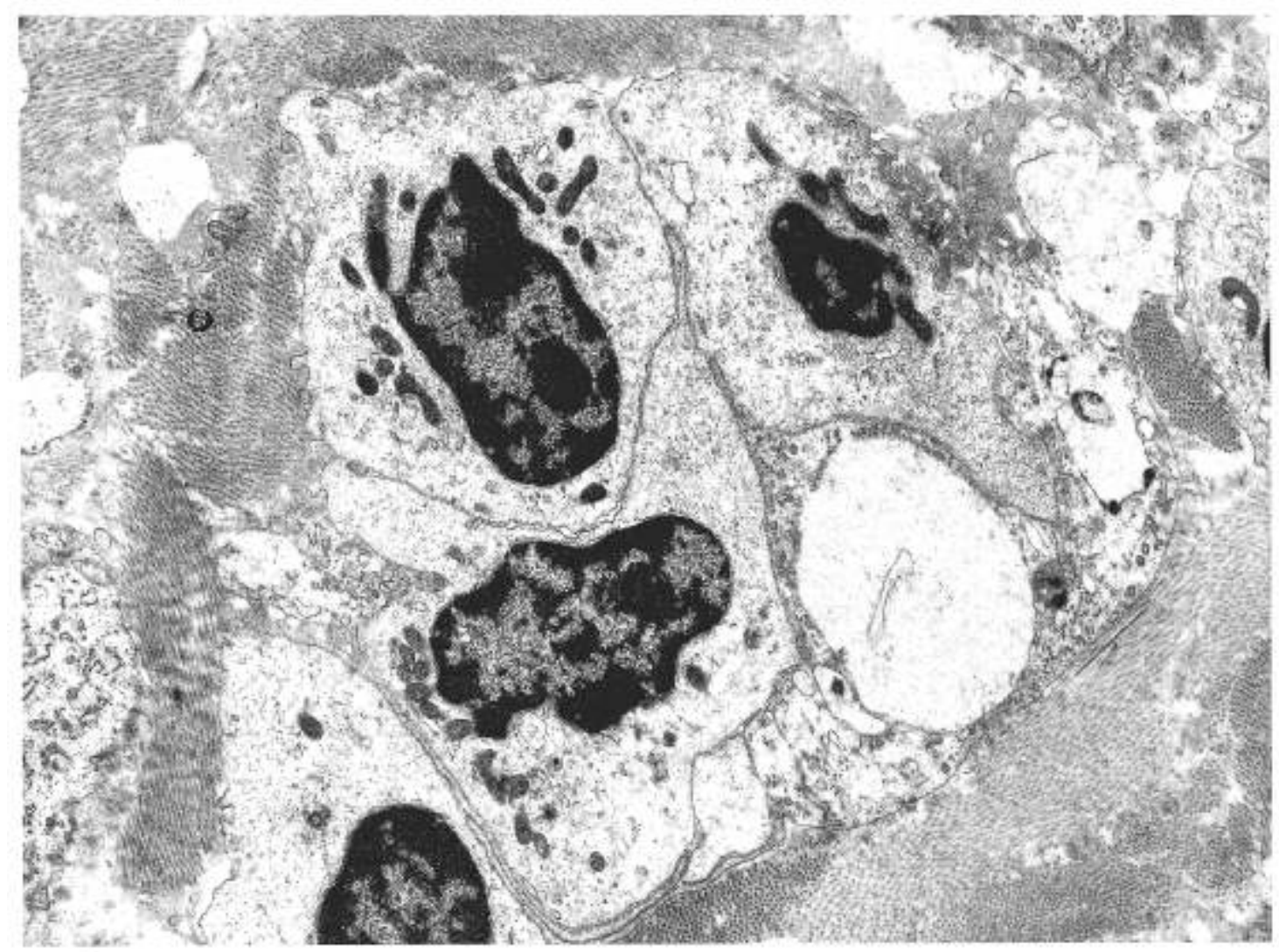

FIG. 10. Electron micrograph showing clusters of rounded cells within spindled areas. Close proximity of cells and interdigitation suggests partial endothelial differentiation.

than most conventional angiosarcomas, the clinical course indicates a potential for extensive growth. Nine of our 14 patients with follow-up information developed additional lesions in the vicinity of the original tumor. However, in two patients the secondary lesions occurred far proximal to the original. These cases raise the question of whether these lesions represent true recurrences in the sense of contiguous regrowth of the primary, whether they represent local metastases occurring hematogenously, or whether they represent separate primaries. In support of the first idea is the fact that two cases occurred within a vessel initially, and it is conceivable that growth along such a structure could give rise to a "scatter" pattern of metastasis. However, careful evaluation of the remaining cases failed to disclose intravenous origin, and examination of minute lesions measuring less than $1 \mathrm{~mm}$ seemed not to support this hypothesis. We also have given serious consideration to the second possibility - that the multiple lesions are, in reality, local metastases. If this were the case, we would have expected to document metastases in conventional locations in many of our cases. Only one patient in our series developed a regional lymph node metastasis. Since that patient had also been irradiated and his tumor showed transition to a high-grade sarcoma, it is difficult to place undue significance on this event. In all probability, then, this is a multifocal disease similar to Kaposi's sarcoma and certain angiosarcomas, particularly those that occur in chronic lymphedema (15). In this regard, it should be noted that one of our patients had Milroy's disease and developed his tumor in an area of chronic lymphedema.

Since this tumor displays many of the clinical features of Kaposi's sarcoma, such as multifocality, acral location on the extremities, and male predominance, the question might be raised as to whether this is, in fact, a form of Kaposi's sarcoma. We believe there are several features which set this tumor apart from Kaposi's sarcoma. First, the presence of large, cavernous vessels filled with organizing thrombi and phleboliths are at variance with Kaposi's sarcoma. This feature was present in virtually all cases and in the earliest lesions actually dominated the histological picture, so that the diagnosis of a cavernous hemangioma was favored. Although the early stage or peripheral zones of $\mathrm{Ka}$ posi's sarcoma may have an angiomatous appearance, the vessels are of capillary, not cavernous size. Second, the epithelioid endothelial cells, 


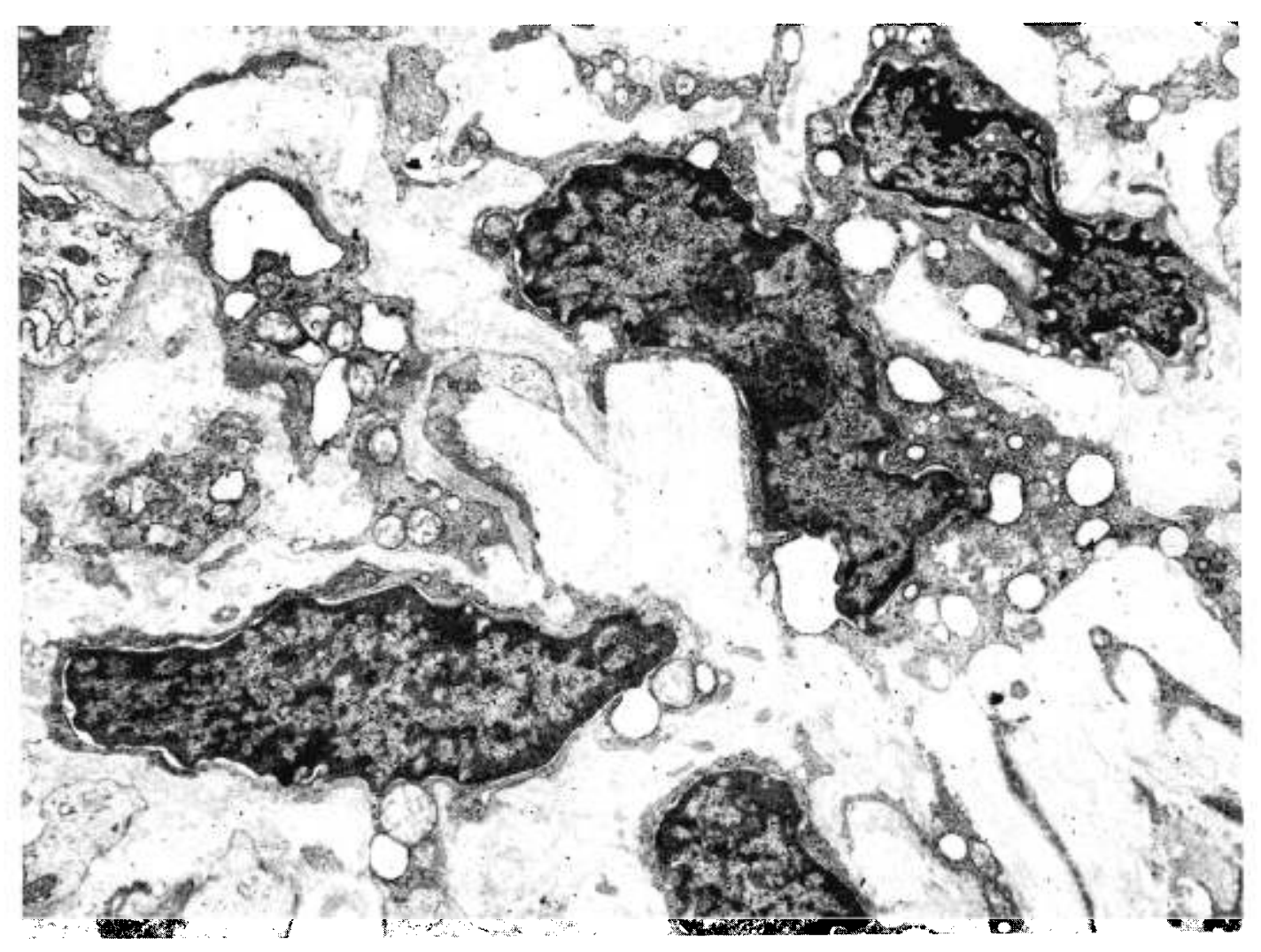

FIG. 11. Electron micrograph of poorly differentiated mesenchymal cells within spindled areas.

which contain immunoreactive factor VIII-associated protein and occasional cytoplasmic "vacuoles" and which lie scattered throughout the spindled stroma, contrast with Kaposi's sarcoma. In the latter tumor the spindled stroma is composed of a single population of uniform-appearing spindle cells (5). Although their exact line of differentiation has been debated, most studies have failed to document features of conventional endothelium. Likewise, the presence of factor VIII-associated protein within the spindled cells of Kaposi's sarcoma has also been controversial $(2,12)$, but a recent critical study of the problem indicates that they do not contain the antigen (11). Third, the presentation of our tumors differs from Kaposi's sarcoma. The young age of the patients in our series contrasts markedly with classic Kaposi's sarcoma in this country. The lack of known risk factors and the ascertainment of cases prior to 1979 (9) argue against the possibility that these are AIDS-related. Nonetheless, we have been impressed by the increasing frequency with which these cases have been referred to us. Thirteen of our 26 cases were received in the past 12 months. In the absence of serologic data, we cannot completely exclude the possibility that these tumors are an expression of infection with HTLV-III. More likely, however, is the possibility that increasing frequency of this tumor in our consultation experience reflects the growing concern within the medical community to correctly diagnose Kaposi's sarcoma. In any event, it is important to point out that the clinical course of SCH is at variance with both classic and AIDSrelated Kaposi's sarcoma. Although two-thirds of the patients developed extensive local disease, no patient has died of the disease even though therapy has been conservative in most instances. However, as with any low-grade sarcoma, long term followup in a large number of patients is required for assessment of metastatic potential.

Biologically these tumors can be placed in the same general category as the "epithelioid hemangioendothelioma" (13) and the "malignant endovascular papillary angioendothelioma" of Dabska (6), two tumors that infrequently give rise to metastatic disease. Interestingly, one of our 26 patients also had an epithelioid hemangioendothelioma adjacent to several SCHs, suggesting a relationship between these low-grade angiosarcomas and opening the possibility that rare transitional forms may yet be documented. Accordingly, we suggest the term "spindle cell hemangioendothelioma" for this vascular tumor of intermediate or low-grade malignancy. Guidelines for therapy of this tumor are 
complicated by the issue of multifocality. Solitary or multiple lesions within a small area are probably best treated by wide local excision without radiotherapy or chemotherapy. We have no data available to suggest whether radiotherapy or chemotherapy would be more effective than surgery in controlling multiple lesions involving a large area of the body. The decision to use radiotherapy in a given patient, in our opinion, depends on the need or urgency to control local disease versus the complications from the therapy. The only patient to develop metastatic disease was one who had been treated with radiotherapy. Because of the association of some vascular tumors with exogenous steroids $(7,8,10)$, the fluctuation of rare vascular tumors with change in hormone status (1), and the presence of estrogen receptor protein on normal endothelium (4), two tumors were studied for several hormone receptors. Neither tumor displayed either estrogen or progesterone receptor protein, but both had high levels of glucocorticoid receptor and one had androgen receptors. The clinical significance of these findings, and, indeed, the significance of hormone receptors in soft tissue tumors in general, is unclear $(3,14)$. However, in no patient in our series was there evidence to suggest altered growth in response changes in hormonal status, and no patients were known to be on exogenous steroids.

Acknowledgment: The authors acknowledge the help of the Division of Immunopathology, under the direction of Dr. John Langloss, for the immunohistologic preparations. We also express our appreciation to Dr. Gary Ellis for review of the manuscript and to Dr. Gary Lanham for his critical comments.

\section{REFERENCES}

1. Boley SJ, Morse WE. Hormonally influenced hemangioma. Arch Surg 1957;74:482-4.

2. Burgdorf WCH, Mukai K, Rosai J. Immunohistochemical identification of factor VIII-related antigen in endothelial cells. Am J Clin Pathol 1981;75:167-71.

3. Chaudhuri PK, Walker MJ, Beattie CW, Das Gupta TK Presence of steroid receptors in human soft tissue sarcomas of diverse histological origin. Cancer Res 1980;40:851-5.

4. Colburn P, Buonassisi V. Estrogen-binding sites in endothelial cell cultures. Science 1978;201:817-9.

5. Cox FH, Helwig EB. Kaposi's sarcoma. Cancer 1959; 12:289-98.

6. Dabska M. Malignant endovascular papillary angioendothelioma of the skin in childhood. Cancer 1969;24:503-10.

7. Dean PJ, Haggitt RC, O'Hara CJ. Malignant epithelioid hemangioendothelioma of the liver in young women: Relationship to oral contraceptive use. Am J Surg Pathol $1985 ; 9: 695-704$.

8. Falk H, Popper H, Thomas LB, Ishak KG. Hepatic angiosarcoma associated with androgenic anabolic steroids. Lancet 1979;24:1120-2.

9. Gottlieb MS, Groopman JE, Weinstein WM, et al. The acquired immunodeficiency syndrome. Ann Intern Med 1983:99:208-20.

10. Hoch-Ligeti C. Angiosarcoma of liver associated with diethylstilbesterol. JAMA 1978;240:1510-1.

11. Millard PR, Heryet AR. An immunohistochemical study of factor VIII-related antigen and Kaposi's sarcoma using polyclonal and monoclonal antibodies. J Pathol 1985;146: 31-8.

12. Nadji M, Morales A, Ziegles-Weissman J, Penneys N. Kaposi's sarcoma: Immunohistologic evidence for an endothelial origin. Arch Pathol Lab Med 1981;105:274-5.

13. Weiss SW, Enzinger FM. Epithelioid hemangioendothelioma: A distinctive vascular tumor often mistaken for a carcinoma. Cancer 1982;50;970-81.

14. Weiss SW, Langloss JM, Shmookler BM, Malawer M, D'Avis J, Stanton R. Estrogen receptor protein in bone and soft tissue sarcomas. Lab Invest (in press).

15. Woodward $\mathrm{AH}$, Ivins JC, Soule EH. Lymphangiosarcoma arising in chronic lymphedematous extremities. Cancer 1972;30:562-72. 